\title{
Lasota-Opial type conditions for periodic problem for systems of higher-order functional differential equations
}

\author{
Sulkhan Mukhigulashvili1, $2^{*}$ (D) and Bedřich Pưža ${ }^{2}$
}

\author{
"Correspondence: \\ smukhig@gmail.com \\ ${ }^{1}$ Institute of mathematics of the \\ Czech Academy of Sciences, Brno, \\ Czech Republic \\ ${ }^{2}$ Faculty of Business and \\ Management, Brno University of \\ Technology, Brno, Czech Republic
}

\begin{abstract}
In the paper we study the question of solvability and unique solvability of systems of the higher-order functional differential equations$$
u_{i}^{\left(m_{i}\right)}(t)=\ell_{i}\left(u_{i+1}\right)(t)+q_{i}(t) \quad(i=\overline{1, n}) \text { for } t \in l:=[a, b]
$$

and

$$
u_{i}^{\left(m_{i}\right)}(t)=F_{i}(u)(t)+q_{0 i}(t) \quad(i=\overline{1, n}) \text { for } t \in l
$$

under the periodic boundary conditions

$$
u_{i}^{(j)}(b)-u_{i}^{(j)}(a)=c_{i j} \quad\left(i=\overline{1, n}, j=\overline{0, m_{i}-1}\right),
$$

where $u_{n+1}=u_{1}, m_{i} \geq 1, n \geq 2, c_{i j} \in R, q_{i}, q_{0 i} \in L(1 ; R), \ell_{i}: C_{1}^{0}(1 ; R) \rightarrow L(1 ; R)$ are monotone operators and $F_{i}$ are the local Caratheodory's class operators. In the paper in some sense optimal conditions that guarantee the unique solvability of the linear problem are obtained, and on the basis of these results the optimal conditions of the solvability and unique solvability for the nonlinear problem are proved.
\end{abstract}

MSC: 34K06; 34K13; 34B05

Keywords: Higher-order systems; Periodic problem; Functional differential equations; Unique solvability

\section{Introduction}

Consider on the interval $I=[a, b]$ the system of higher-order linear functional differential equations

$$
u_{i}^{\left(m_{i}\right)}(t)=\ell_{i}\left(u_{i+1}\right)(t)+q_{i}(t) \quad(i=\overline{1, n})
$$

(c) The Author(s) 2020. This article is licensed under a Creative Commons Attribution 4.0 International License, which permits use, sharing, adaptation, distribution and reproduction in any medium or format, as long as you give appropriate credit to the original author(s) and the source, provide a link to the Creative Commons licence, and indicate if changes were made. The images or other third party material in this article are included in the article's Creative Commons licence, unless indicated otherwise in a credit line to the material. If material is not included in the article's Creative Commons licence and your intended use is not permitted by statutory regulation or exceeds the permitted use, you will need to obtain permission directly from the copyright holder. To view a copy of this licence, visit http://creativecommons.org/licenses/by/4.0/. 
where $u_{n+1}:=u_{1}$, and the system of higher-order nonlinear functional differential equations

$$
u_{i}^{\left(m_{i}\right)}(t)=F_{i}(u)(t)+q_{0 i}(t) \quad(i=\overline{1, n})
$$

where $m_{i} \geq 1, q_{i}, q_{0 i} \in L(I ; R), \ell_{i}: C_{1}^{0}(I ; R) \rightarrow L(I ; R)$ are linear bounded operators, and $F_{i} \in K\left(C^{m_{1}, \ldots, m_{n}}, L\right)$ (see Definition 1.1), under the periodic boundary conditions

$$
u_{i}^{(j)}(b)-u_{i}^{(j)}(a)=c_{i j} \quad\left(i=\overline{1, n}, j=\overline{0, m_{i}-1}\right)
$$

Throughout the paper we use the following notations: $N$ is the set of the natural numbers; $R=]-\infty,+\infty\left[, R_{+}=\left[0,+\infty\left[; R^{n}\right.\right.\right.$ is the space of the $n$th dimensional column vectors $x:=\left(x_{i}\right)_{i=1}^{n}$ with the components $x_{i} \in R(i=\overline{1, n})$ and the norm $\|x\|=\sum_{i=1}^{n}\left|x_{i}\right| ; C_{1}^{n-1}(I ; R)$ $(n \in N)$ is the Banach space of the functions $u: I \rightarrow R$ which are continuous together with their $(n-1)$ th derivatives, with the norm

$$
\|u\|_{C_{1}^{n-1}}=\max \left\{\sum_{j=1}^{n}\left|u^{(j-1)}(t)\right|: t \in I\right\} ;
$$

$C^{m_{1}, \ldots, m_{n}}\left(I ; R^{n}\right)$ is the Banach space of the vector-functions $u:=\left(u_{i}\right)_{i=1}^{n}: I \rightarrow R^{n}$, where $u_{i} \in$ $C_{1}^{m_{i}-1}(I ; R)(i=\overline{1, n})$, with the norm

$$
\|u\|_{C^{m_{1}, \ldots, m_{n}}}=\max \left\{\sum_{i=1}^{n} \sum_{j=1}^{m_{i}}\left|u_{i}^{(j-1)}(t)\right|: t \in I\right\},
$$

and for the case when $m_{i}=1(i=\overline{1, n})$, we will use the notations

$$
C_{n}^{0}\left(I ; R^{n}\right):=C^{1, \ldots, 1}\left(I ; R^{n}\right), \quad\|u\|_{C_{n}^{0}}:=\|u\|_{C^{1, \ldots, 1}} ;
$$

$\widetilde{C}^{0}(I ; R)$ is the Banach space of the functions $u: I \rightarrow R$ which are absolutely continuous with the norm $\|u\|_{\widetilde{C}^{0}}=\|u\|_{C}+\int_{a}^{b}\left|u^{\prime}(s)\right| d s ; \widetilde{C}^{n-1}(I ; R)(n \in N)$ is a set of functions $u: I \rightarrow R$ which are absolutely continuous together with their $(n-1)$ th derivatives; $L(I ; R)$ is the Banach space of the Lebesgue integrable functions $p: I \rightarrow R$ with the norm $\|p\|_{L}=$ $\int_{a}^{b}|p(s)| d s ; M^{n}(I)$ is the set of the vector-functions $\tau:=\left(\tau_{i}\right)_{i=1}^{n}: I \rightarrow I^{n}$, with the measurable components $\tau_{i}: I \rightarrow I(i=\overline{1, n})$.

For arbitrary $x \in R$, we assume that

$$
\operatorname{sgn} x= \begin{cases}1 & \text { if } x \geq 0 \\ -1 & \text { if } x<0\end{cases}
$$

Definition 1.1 We will say that the operator $F: C^{m_{1}, \ldots, m_{n}}\left(I ; R^{n}\right) \rightarrow L(I ; R)$ belongs to Caratheodory's local class $K\left(C^{m_{1}, \ldots, m_{n}}, L\right)$ if $F$ is a continuous operator, and for arbitrary $r>0$, the inclusion

$$
\sup \left\{|F(x)(\cdot)|:\|x\|_{C^{m_{1}, \ldots, m_{n}}} \leq r, x \in C^{m_{1}, \ldots, m_{n}}\left(I ; R^{n}\right)\right\} \in L\left(I ; R_{+}\right)
$$

holds. 
Definition 1.2 We will say that a linear operator $\ell: C_{1}^{0}(I ; R) \rightarrow L(I ; R)$ is nonnegative (nonpositive) if, for any nonnegative $x \in C_{1}^{0}(I ; R)$, the inequality $\ell(x)(t) \geq 0(\ell(x)(t) \leq 0)$ for $t \in I$ is satisfied.

We will say that an operator $\ell$ is monotone if it is nonnegative or nonpositive.

By a solution of problem (2), (3) we understand a vector-function $u:=\left(u_{i}\right)_{i=1}^{n}$ where $u_{i} \in \widetilde{C}^{m_{i}-1}(I ; R)(i=\overline{1, n})$, which satisfies equation (2) almost everywhere on $I$ and satisfies conditions (3).

First of all, we would like to give a historical review which begins with Lasota and Opial's article [1] from the year 1964, which became the basis for a lot of interesting studies. In this article (see Theorems 5 and 6) authors proved that the problem

$$
\begin{aligned}
& u^{(n)}(t)=p(t) u(t)+q(t) \quad \text { for } t \in I, \\
& u^{(j)}(b)-u^{(j)}(a)=c_{j} \quad(j=\overline{0, n-1}),
\end{aligned}
$$

is uniquely solvable if $\int_{a}^{b} p(s) d s \leq 0$ for $n=2, p(t) \leq 0$ for $n \geq 3$, and

$$
\int_{a}^{b}|p(s)| d s<\frac{L_{n}}{(b-a)^{n-1}}, \quad \text { and } \quad p \not \equiv 0
$$

where $L_{2}=16$ and the general term $L_{n}$ can be found by formula (40) from the paper [1], in which it is also shown that for $n=2$ condition (5) is optimal, while for $n \geq 3$ it is far from being optimal.

In the article [2], we announced the Lasota-Opial type optimal results of unique solvability of the periodic problem for second-order linear functional differential equations, which in a more general form are considered in the paper [3] from 2006. In particular, in Theorem 1.1 of the paper [3] (see also [4]) it is proved that the functional differential equation

$$
u^{(n)}(t)=\sum_{i=0}^{n-1} \ell_{i}\left(u^{(i)}\right)(t)+q(t)
$$

under boundary conditions (4) for $n=2$ is uniquely solvable if the optimal conditions

$$
\int_{a}^{b} \ell_{0}(1)(s) d s \neq 0
$$

and

$$
\int_{a}^{b}\left|\ell_{0}(1)(s)\right| d s \leq \frac{16}{b-a}\left(1-\int_{a}^{b}\left(\ell_{1}^{+}(1)(s)+\ell_{1}^{-}(1)(s)\right) d s\right)
$$

hold, where $\ell_{0}: C_{1}^{0}(I ; R) \rightarrow L(I ; R)$ is a monotone operator, $\ell_{i}=\ell_{i}^{+}-\ell_{i}^{-}(i=\overline{1, n-1})$ and $\ell_{i}^{ \pm}: C_{1}^{0}(I ; R) \rightarrow L(I ; R)$ are nonnegative operators (for $n=3$ see [5]). For the general case, analogous conditions of the unique solvability of problem (6), (4) are proved in [6], for $\ell_{i}=$ $\ell_{i}^{+}-\ell_{i}^{-}(i=\overline{0, n-1})$, which if $\ell_{0}$ is monotone operator and $\ell_{i} \equiv 0(i=\overline{1, n-1})$ transforms 
to the conditions

$$
\int_{a}^{b} \ell_{0}(1)(s) d s \neq 0 \quad \text { and } \quad \int_{a}^{b}\left|\ell_{0}(1)(s)\right| d s \leq \frac{4 T_{n-1}}{(b-a)^{n-1}}
$$

where $T_{1}=4, T_{2}=32, T_{3}=192$, and the general term $T_{n}$ of this sequence can be found by formulas (9). In [6] (see also [7]) it is proved that the constants $T_{n}$ are sharp when $n \leq 7$; for $n>7$, the problem of sharpness of the numbers $T_{n}$ is still opened.

In this brief historical review Bravyi's original studies of problem (6), (4) cannot be omitted. The method developed in these studies turned out to be very fruitful. Particularly in [8] the author proved that the condition

$$
\frac{Y}{1-Y} \leq X \leq 2(1+\sqrt{1-Y})
$$

where $Y=N_{n}(b-a)^{n-1} \min \left(P^{-}, P^{+}\right), X=N_{n}(b-a)^{n-1} \max \left(P^{-}, P^{+}\right)$, the numbers $N_{n}$ are defined by the certain recurrent formula, and $\left\|\ell_{0}^{+}(1)\right\|_{L}=P^{+},\left\|\ell_{0}^{-}(1)\right\|_{L}=P^{-}$, is necessary and sufficient for solvability of problems (6), (4) if $\ell_{i} \equiv 0(i=\overline{1, n-1})\left(N_{n}=T_{n-1}^{-1}\right.$ for $n=\overline{2,7}$, but for $n>7$ the validity of the last identity is unknown). For the case $\ell_{i} \not \equiv 0(i=\overline{1, n-1})$, these results are generalized in [9]. It is interesting that the numbers $N_{n}$ are in some connection with Favard's, Bernoulli's, and Euler's numbers (see [10] and [11]) and if the operator $\ell_{0}$ is monotone, then condition (8) transforms to the condition $0<\int_{a}^{b}\left|\ell_{0}(1)(s)\right| d s \leq \frac{4}{N_{n}(b-a)^{n-1}}$. Other interesting results about problems (6), (4) can be found also in the papers [10-15].

The next stage was the generalization of Lasota-Opial's results for the systems of linear functional differential equations. In particular in [16] it is proved that problem (1), (3) with $m_{i}=1(i=\overline{1, n})$ is uniquely solvable if $\ell_{i}$ are linear monotone operators, $\left\|\ell_{i}\right\| \neq 0(i=\overline{1, n})$, and the condition $\prod_{i=1}^{n}\left\|\ell_{i}(1)\right\|_{L}<4^{n}$ (which is optimal) holds. In this connection see also the papers [17] and [18].

The aims of this article are to establish Lasota-Opial type sufficient efficient optimal conditions of the solvability of problem (1), (3) and on the basis of these results to find the optimal efficient sufficient conditions of solvability and unique solvability of the nonlinear problem (2), (3).

\section{Main results}

\subsection{Linear problem}

Let $T_{0}=1, T_{1}=4, T_{2}=32, T_{3}=192$, and

$$
\begin{aligned}
T_{2 m+2} & =\frac{1}{\max \left\{\left(h_{m}(t) h_{m}(1-t)\right)^{1 / 2}: 0 \leq t \leq 1\right\}}, \\
T_{2 m+3} & =\frac{1}{\max \left\{\left(f_{m}(t, s) f_{m}(1-t, 1-s)\right)^{1 / 2}: 0 \leq t \leq 1,0 \leq s \leq 1\right\}}
\end{aligned}
$$

for $m \geq 1$, where the functions $f_{m}:[0,1] \times[0,1] \rightarrow R_{+}, h_{m}:[0,1] \rightarrow R_{+}$are defined by the equalities

$$
f_{m}(t, s)=\sum_{j=0}^{m-1} \alpha_{m j} t^{2(j+1)}+\alpha_{m m} s^{2 m+3}, \quad h_{m}(t)=\sum_{j=0}^{m} \beta_{m j} t^{2(j+1)},
$$


where

$$
\begin{aligned}
& \alpha_{m j}=\frac{A_{j}}{3 \cdot 4^{j+1} T_{2(m-j)+1}}, \quad \beta_{m j}=\frac{A_{j}}{3 \cdot 4^{j+1} T_{2(m-j)}} \quad \text { for } j=\overline{0, m-1}, \\
& \alpha_{m m}=\frac{A_{m}}{3 \cdot 4^{m+1}}, \quad \beta_{m m}=\frac{B_{m}}{3 \cdot 4^{m+1}},
\end{aligned}
$$

and

$$
\begin{aligned}
& A_{0}=1, \quad A_{1}=\frac{1}{15}, \quad A_{j}=A_{1} \sum_{m_{1}=1}^{2} \sum_{m_{2}=1}^{m_{1}+1} \ldots \sum_{m_{j-1}=1}^{m_{j-2}+1} \frac{1}{\eta\left(m_{1}\right) \ldots \eta\left(m_{j-1}\right)}, \\
& B_{1}=\frac{1}{8}, \quad B_{j}=A_{1} \sum_{m_{1}=1}^{2} \sum_{m_{2}=1}^{m_{1}+1} \ldots \sum_{m_{j-1}=1}^{m_{j-2}+1}\left(\frac{1}{\eta\left(m_{1}\right) \ldots \eta\left(m_{j-1}\right)} \prod_{i=1}^{m_{j-1}+1}\left(1+\frac{1}{2 i}\right)\right)
\end{aligned}
$$

for $j \geq 2$, with $\eta(t)=(2 t+1)(2 t+3)$.

Remark 2.1 In Remarks 1.2 and 1.3 of [7] it was shown that

$$
T_{4}=\frac{2^{11} \cdot 3}{5}, \quad T_{5}=2^{9} \cdot 3 \cdot 5, \quad T_{6}=\frac{2^{16} \cdot 3^{2} \cdot 5}{61}, \quad T_{7}=\frac{2^{14} \cdot 3^{2} \cdot 5 \cdot 7}{17},
$$

and

$$
T_{n}<(2 \pi)^{n} \quad(n \in N)
$$

Now we can formulate the first of our main theorems.

Theorem 2.1 Let the operators $\ell_{i}: C_{1}^{0}(I ; R) \rightarrow L(I ; R)(i=\overline{1, n})$ be monotone,

$$
\int_{a}^{b} \ell_{i}(1)(s) d s \neq 0 \quad(i=\overline{1, n})
$$

and the condition

$$
\prod_{i=1}^{n} \frac{1}{T_{m_{i}-1}} \int_{a}^{b}\left|\ell_{i}(1)(s)\right| d s \leq 4^{n}(b-a)^{n-\sum_{i=1}^{n} m_{i}}
$$

hold. Then problem (1), (3) is uniquely solvable.

For the system

$$
u_{i}^{\left(m_{i}\right)}(t)=p_{i}(t) u_{i+1}\left(\tau_{i}(t)\right)+q_{i}(t) \quad(i=\overline{1, n}),
$$

where $u_{n+1}:=u_{1}, \tau:=\left(\tau_{i}\right)_{i=1}^{n} \in M^{n}(I)$, and $p_{i}, q_{i} \in L(I ; R)$, from Theorem 2.1 we have the following.

Corollary 2.1 Let the function $p \in L\left(I ; R^{n}\right)$ be such that the conditions

$$
\int_{a}^{b} p_{i}(s) d s \neq 0, \quad 0 \leq \sigma_{i} p_{i}(t) \quad(i=\overline{1, n}) \text { for } t \in I
$$


where $\sigma_{i} \in\{-1,1\}(i=\overline{1, n})$, and

$$
\prod_{i=1}^{n} \frac{1}{T_{m_{i}-1}} \int_{a}^{b}\left|p_{i}(s)\right| d s \leq 4^{n}(b-a)^{n-\sum_{i=1}^{n} m_{i}}
$$

hold. Then, for arbitrary $\tau \in M^{n}(I)$, problem (12), (3) is uniquely solvable.

Remark 2.2 For some values of the numbers $n, m_{1}, \ldots, m_{n}$, condition (11) is optimal. For example, if $n=2$ and $n=2, m_{1}=m_{2}=1$, then problem (1), (3) with $\ell_{1}(u) \equiv u, \ell_{2}=\ell_{0}$, $q_{1} \equiv 0$ transforms to problem (6), (4) with $\ell_{1} \equiv 0, q \equiv q_{2}$ and condition (11) transforms to condition (7) which, as we have already said, is optimal for the unique solvability of problem (6),(4) with $n=2$.

\subsection{Nonlinear problem}

Definition 2.1 Let $h_{i}: C_{1}^{0}(I ; R) \rightarrow L(I ; R)(i=\overline{1, n})$ be nonnegative linear operators, then we will say that

$$
h:=\left(h_{i}\right)_{i=1}^{n} \in P(I)
$$

if

$$
\int_{a}^{b} h_{i}(1)(s) d s \neq 0 \quad(i=\overline{1, n})
$$

and for arbitrary monotone operators $\ell_{i}: C_{1}^{0}(I ; R) \rightarrow L(I ; R)(i=\overline{1, n})$ such that the conditions

$$
\int_{a}^{b} \ell_{i}(1)(s) d s \neq 0, \quad 0 \leq \sigma_{i} \ell_{i}(1)(t) \leq h_{i}(1)(t) \quad(i=\overline{1, n}) \text { for } t \in I
$$

hold, where $\sigma_{i} \in\{-1,1\}(i=\overline{1, n})$, the homogeneous problem

$$
\begin{aligned}
& u_{i}^{\left(m_{i}\right)}(t)=\ell_{i}\left(u_{i+1}\right)(t) \quad(i=\overline{1, n}), \\
& u_{i}^{(j)}(b)-u_{i}^{(j)}(a)=0 \quad\left(i=\overline{1, n}, j=\overline{0, m_{i}-1}\right)
\end{aligned}
$$

where $u_{n+1}:=u_{1}$, has no nontrivial solution.

Also note that in all our propositions below the functions $\eta_{i}: I \times R_{+} \rightarrow R_{+}(i=\overline{1, n})$ are summable in the first argument, nondecreasing in the second one, and admit to the conditions

$$
\lim _{\rho \rightarrow+\infty} \frac{1}{\rho} \int_{a}^{b} \eta_{i}(s, \rho) d s=0 \quad(i=\overline{1, n}) .
$$

Theorem 2.2 Let the linear nonnegative operators $h_{i}: C_{1}^{0}(I ; R) \rightarrow L(I ; R)(i=\overline{1, n})$, the function $g_{0} \in L\left(I ; R^{n}\right)$, and the numbers $\sigma_{i} \in\{-1,1\}(i=\overline{1, n}), r_{0}>0$, be such that, for all 
$i \in\{1, \ldots, n\}$ on $I$, the conditions

$$
\begin{aligned}
& g_{0 i}(t) \leq \sigma_{i} F_{i}(x)(t) \operatorname{sgn} h_{i}\left(x_{i+1}\right)(t) \leq\left|h_{i}\left(x_{i+1}\right)(t)\right|+\eta_{i}\left(t,\|x\|_{C^{m_{1}, \ldots, m_{n}}}\right) \\
& \text { if }\|x\|_{C^{m_{1}, \ldots, m_{n}}} \geq r_{0},
\end{aligned}
$$

with $x:=\left(x_{i}\right)_{i=1}^{n} \in C^{m_{1}, \ldots, m_{n}}\left(I ; R^{n}\right), x_{n+1}=x_{1}$, and inclusion (13) hold. Moreover, let the function $g \in L\left(I ; R_{+}^{n}\right)$ be such that, for all $i \in\{1, \ldots, n\}$ on $I$, the conditions

$$
g_{i}(t) \leq \sigma_{i} F_{i}(x)(t) \operatorname{sgn} h_{i}\left(x_{i+1}\right)(t) \quad \text { if } \min \left\{\left|x_{i+1}(t)\right|: t \in I\right\} \geq r_{0}
$$

are fulfilled, and

$$
\int_{a}^{b} g_{i}(s) d s-\left|\int_{a}^{b} q_{0 i}(s) d s\right| \geq\left|c_{i m_{i}-1}\right|
$$

Then problem (2), (3) has at least one solution.

Remark 2.3 From the inequality $\|x\|_{C^{m_{1}, \ldots, m_{n}}} \geq \min \left\{\left|x_{i+1}(t)\right|: t \in I\right\}$ it follows that $g_{0 i} \leq g_{i}$. Therefore, if we assume that instead of (21) inequalities

$$
\int_{a}^{b} g_{0 i}(s) d s-\left|\int_{a}^{b} q_{0 i}(s) d s\right| \geq\left|c_{i m_{i}-1}\right| \quad(i=\overline{1, n})
$$

hold, then condition (20) can be omitted.

On the other hand, in the example below we construct the operator $F$ for which conditions (21) hold and (22) do not hold, and therefore condition (20) cannot be omitted because, as it follows from Remark 2.4, condition (21) is improvable.

Example 2.1 Consider system (2) with

$$
F_{i}(u)(t)= \begin{cases}\sigma_{i} \widetilde{h}_{i}(t) \cos \left(\frac{\pi y}{\left(x_{i+1}(t)+y\right) x_{i+1}(t)}\right) x_{i+1}(t) & \text { if } x_{i+1}(t) \neq 0 \\ 0 & \text { if } x_{i+1}(t)=0\end{cases}
$$

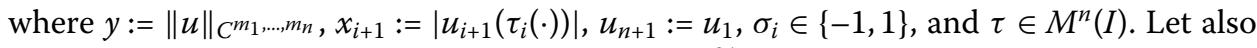
the numbers $\delta_{1} \in(0,1), c_{i m_{i}-1}$ and the functions $\widetilde{h} \in L\left(I ; R_{+}^{n}\right), q_{0} \in L\left(I ; R^{n}\right)$ be such that inclusion (13) with $h_{i}(z)(\cdot)=\widetilde{h}_{i}(\cdot) z\left(\tau_{i}(\cdot)\right)$ holds, and

$$
\left(\int_{a}^{b} \tilde{h}_{i}(s) d s\right)^{-1}\left(\left|\int_{a}^{b} q_{0 i}(s) d s\right|+\left|c_{i m_{i}-1}\right|\right) \leq \delta_{1} \quad(i=\overline{1, n})
$$

Then, if $\alpha(x)=x \cos (\pi / x)$, in view the facts that

$$
\alpha(2)=0, \quad \alpha^{\prime}(x)>0 \quad \text { if } x>2, \quad \text { and } \quad \lim _{x \rightarrow+\infty} \alpha^{\prime}(x)=1
$$

there exist $r_{0}>2$ and $\delta_{0} \in\left(0, r_{0}\right)$ such that $r_{0} \cos \left(\pi / r_{0}\right)=\delta_{1}$, and the inequalities

$$
\begin{aligned}
& \delta_{1} \leq \cos \left(\frac{\pi}{x_{i+1}(t)}\right) x_{i+1}(t)<\cos \left(\frac{\pi y}{\left(x_{i+1}(t)+y\right) x_{i+1}(t)}\right) x_{i+1}(t) \\
& \quad \text { if } x_{i+1}(t) \geq r_{0}, y \geq r_{0},
\end{aligned}
$$


and

$$
\begin{aligned}
& -\delta_{0} \leq \cos \left(\frac{\pi y}{\left(x_{i+1}(t)+y\right) x_{i+1}(t)}\right) x_{i+1}(t) \leq x_{i+1}(t) \\
& \quad \text { if } 0<x_{i+1}(t)<r_{0}, y \geq r_{0},
\end{aligned}
$$

are valid on $I$. Therefore, the validity of conditions (19)-(21) with $g_{i} \equiv \delta_{1} \widetilde{h}_{i}, g_{0 i} \equiv-\delta_{0} \widetilde{h}_{i}$, $\eta_{i} \equiv 0$ is obvious, and from Theorem 2.2 the solvability of problem (2), (3) follows.

Corollary 2.2 Let the linear nonnegative operators $h_{i}: C_{1}^{0}(I ; R) \rightarrow L(I ; R)(i=\overline{1, n})$ be such that conditions (14) and

$$
\prod_{i=1}^{n} \frac{1}{T_{m_{i}-1}} \int_{a}^{b} h_{i}(1)(s) d s \leq 4^{n}(b-a)^{n-\sum_{i=1}^{n} m_{i}}
$$

hold. Moreover, let the functions $g_{0} \in L\left(I ; R^{n}\right), g \in L\left(I ; R_{+}^{n}\right)$ and the numbers $\sigma_{i} \in\{-1,1\}$ $(i=\overline{1, n}), r_{0}>0$ be such that conditions (19)-(21) are fulfilled. Then problem (2), (3) has at least one solution.

For the case when (2) is the system of higher-order differential equations with the argument deviation of the form

$$
u_{i}^{\left(m_{i}\right)}(t)=f_{i}\left(t, u_{i+1}\left(\tau_{i}(t)\right)\right)+q_{0 i}(t) \quad(i=\overline{1, n}),
$$

where $u_{n+1}:=u_{1}$ and $f_{i}: I \times R \rightarrow R(i=\overline{1, n})$ are the functions from Caratheodory's class, the following corollary is true.

Corollary 2.3 Let the function $\widetilde{h} \in L\left(I ; R_{+}^{n}\right)$ be such that the conditions

$$
\begin{aligned}
& \widetilde{h}_{i}(t)>0 \quad(i=\overline{1, n}) \text { a.e.on } I, \\
& \prod_{i=1}^{n} \frac{1}{T_{m_{i}-1}} \int_{a}^{b} \tilde{h}_{i}(s) d s \leq 4^{n}(b-a)^{n-\sum_{i=1}^{n} m_{i}}
\end{aligned}
$$

hold. Moreover, let the function $g \in L\left(I ; R_{+}^{n}\right)$ and the numbers $\sigma_{i} \in\{-1,1\}(i=\overline{1, n}), r_{0}>0$ be such that conditions (21) and

$$
\begin{aligned}
& g_{i}(t) \leq \sigma_{i} f_{i}(t, x) \operatorname{sgn} x \leq \tilde{h}_{i}(t)|x|+\eta_{i}(t,|x|) \\
& \quad \text { for }|x| \geq r_{0}, t \in I(i=\overline{1, n}),
\end{aligned}
$$

hold. Then, for arbitrary $\tau \in M^{n}(I)$, problem (24), (3) has at least one solution.

Remark 2.4 Theorem 2.2 is optimal in the sense that there does not exist such $i_{0} \in$ $\{1, \ldots, n\}$, for which $i_{0}$ th inequality of condition (21) can be replaced by the inequality

$$
\varepsilon+\int_{a}^{b} g_{i_{0}}(s) d s-\left|\int_{a}^{b} q_{0 i_{0}}(s) d s\right| \geq\left|c_{i_{0} m_{i_{0}}-1}\right|
$$


no matter how small $\varepsilon>0$ would be. Indeed, let $I=[0,1]$,

$$
\begin{aligned}
& \sigma_{i} \in\{-1,1\}, \quad m_{i}=1, \quad c_{i 0}=0, \quad \tau_{i} \equiv t, \quad \eta_{i} \equiv 0 \quad(i=\overline{1, n}), \\
& \widetilde{h}_{i} \equiv 1, \quad g_{i} \equiv 1, \quad q_{0 i} \equiv 0, \quad f_{i}(t, x) \equiv \sigma_{i} x \quad\left(i=\overline{1, n} ; i \neq i_{0}\right),
\end{aligned}
$$

and

$$
g_{i_{0}} \equiv 0, \quad q_{0 i_{0}}=\frac{\varepsilon}{1+\varepsilon}, \quad f_{i_{0}}(t, x) \equiv 0, \quad \widetilde{h}_{i_{0}} \equiv 4^{n}-\varepsilon
$$

Then, for the arbitrary functions $p_{i} \in L(I ; R)$ such that $p_{i} \neq \equiv 0,0 \leq \sigma_{i} p_{i}(t) \leq \widetilde{h}_{i}(t)$, it is clear that $\prod_{i=1}^{n} \int_{a}^{b}\left|p_{i}(s)\right| d s \leq 4^{n}$, and due to Corollary 2.1 inclusion (13) with $h_{i}(x)(t)=$ $\widetilde{h}_{i}(t) x\left(\tau_{i}(t)\right)$ holds. Also it is not difficult to verify that instead of the $i_{0}$ th inequality of condition (21) inequality (27) is satisfied, but all the other assumptions of Corollary 2.3 hold with $r_{0}=1$. Nevertheless, in that case problem (24), (3) is not solvable because $u_{i_{0}}(1)-u_{i_{0}}(0)=\varepsilon /(1+\varepsilon)>0=c_{i_{0}}$.

Example 2.2 Consider the system of the differential equations

$$
u_{i}^{\left(m_{i}\right)}(t)=\sigma_{i} \widetilde{h}_{i}(t)\left|\sin \|u\|_{C^{m_{1}, \ldots, m_{n}}}\right| u_{i+1}\left(\tau_{i}(t)\right)+q_{0 i}(t) \quad(i=\overline{1, n})
$$

where $u_{n+1}=u_{1}, \sigma_{i} \in\{-1,1\}$, the functions $\tau \in M^{n}(I), \tilde{h} \in L\left(I ; R_{+}^{n}\right)$ are such that inclusion (13) holds with $h_{i}(x)(t)=\widetilde{h}_{i}(t) x\left(\tau_{i}(t)\right)$, and $\int_{a}^{b} q_{0 i}(s) d s=0(i=\overline{1, n})$. Then, from Theorem 2.2 with $g_{0 i} \equiv g_{i} \equiv 0$, the solvability of problems (28), (3) with $c_{i m_{i}-1}=0(i=\overline{1, n})$ follows.

Also, on the basis of Corollary 2.3, we can prove the following existence and uniqueness theorem.

Theorem 2.3 Let the function $\widetilde{h} \in L\left(I ; R_{+}^{n}\right)$ be such that conditions (25) hold and $f_{i}(t, 0) \equiv$ $0(i=\overline{1, n})$. Moreover, let the functions $\widetilde{h}_{0} \in L\left(I ; R_{+}^{n}\right), \beta_{i}: R^{2} \rightarrow R_{+}$and the numbers $\sigma_{i} \in$ $\{-1,1\}, r>0$ be such that, for all $i \in\{1, \ldots, n\}$, conditions (21),

$$
\begin{aligned}
& \widetilde{h}_{0 i}(t) \beta_{i}(x, y) \leq \sigma_{i}\left(f_{i}(t, x)-f_{i}(t, y)\right) \operatorname{sgn}(x-y) \leq \widetilde{h}_{i}(t)|x-y| \\
& \quad \text { for } t \in I, x, y \in R
\end{aligned}
$$

and

$$
\widetilde{h}_{0 i}(t) \geq 0 \quad \text { for } t \in I, \quad \widetilde{h}_{0 i}(t) \neq 0, \quad \beta_{i}(x, y)>0 \quad \text { for } x \neq y
$$

hold, where

$$
g_{i}(t)=\min \left\{\left|f_{i}(t, r)\right|,\left|f_{i}(t,-r)\right|\right\} .
$$

Then, for arbitrary $\tau \in M^{n}(I)$, problem (24), (3) is uniquely solvable. 
Example 2.3 As an example, consider the system of differential equations

$$
u_{i}^{\left(m_{i}\right)}(t)=\sigma_{i} \widetilde{h}_{i}(t) \frac{u_{i+1}\left(\tau_{i}(t)\right)}{\left(1+\left|u_{i+1}\left(\tau_{i}(t)\right)\right|\right)^{\alpha_{i}}}+q_{0 i}(t) \quad(i=\overline{1, n})
$$

where $u_{n+1}=u_{1}, \sigma_{i} \in\{-1,1\}$, and the functions $\tau \in M^{n}(I), \widetilde{h} \in L\left(I ; R_{+}^{n}\right)$ are such that inclusion (13) with $h_{i}(x)(t)=\widetilde{h}_{i}(t) x\left(\tau_{i}(t)\right)$ holds, $\alpha_{i} \in(0,1)$, and $\int_{a}^{b} q_{0 i}(s) d s=0(i=\overline{1, n})$. Then from Theorem 2.3 the unique solvability of problem (32), (17) follows.

\section{Auxiliary propositions}

First we formulate a result from [7] (see Theorem 1.1, and Remark 1.1) in a suitable for us form.

Lemma 3.1 Let $m \geq 1, v \in \widetilde{C}^{m}([a, b])$, and the conditions

$$
v(t) \not \text { const }, \quad v^{(i)}(a)=v^{(i)}(b) \quad(i=\overline{0, m})
$$

hold. Then

$$
\triangle(v)<\frac{(b-a)^{m}}{T_{m}} \triangle\left(v^{(m)}\right)
$$

where the constants $T_{m}$ are defined by equalities (9) and

$$
\triangle\left(v^{(i)}\right):=\max _{t \in[a, b]} v^{(i)}(t)-\min _{t \in[a, b]} v^{(i)}(t) \quad(i=\overline{0, m}) .
$$

Lemma 3.2 Let $\sigma \in\{-1,1\}$, the operator $\sigma \ell: C_{1}^{0}(I ; R) \rightarrow L(I ; R)$ be nonnegative, and $w \in$ $C_{1}^{0}(I ; R)$. Then on I the following inequality holds:

$$
\min _{t \in[a, b]}\{w(t)\} \cdot|\ell(1)(t)| \leq \sigma \ell(w)(t) \leq \max _{t \in[a, b]}\{w(t)\} \cdot|\ell(1)(t)| .
$$

Proof Validity of (35) follows from the inequalities $\max _{t \in[a, b]}\{w(t)\}-w(t) \geq 0, w(t)-$ $\min _{t \in[a, b]}\{w(t)\} \geq 0$ and the nonnegativity of the linear operator $\sigma \ell$.

Lemma 3.3 Let the linear monotone operators $h, \ell_{k}: C_{1}^{0}(I ; R) \rightarrow L(I ; R)(k \in N)$ and the number $\sigma \in\{-1,1\}$ be such that $\sigma \ell_{k}$ are nonnegative operators, and

$$
0 \leq \sigma \ell_{k}(1)(t) \leq h(1)(t) \quad(k \in N) \text { for } t \in I .
$$

Then there exist a subsequence $\left(\ell_{k_{r}}\right)_{r=1}^{\infty}$ of the sequence $\left(\ell_{k}\right)_{k=1}^{\infty}$ and the linear operator $\ell_{0}$ : $C_{1}^{0}(I ; R) \rightarrow L(I ; R)$ such that if the operators $\widetilde{\ell}_{k}, \widetilde{\ell}_{0}: C_{1}^{0}(I ; R) \rightarrow \widetilde{C}^{0}(I ; R)$ are defined as

$$
\tilde{\ell}_{k}(x)(t):=\int_{a}^{t} \ell_{k}(x)(s) d s, \quad \tilde{\ell}_{0}(x)(t):=\int_{a}^{t} \ell_{0}(x)(s) d s,
$$

then

$$
\lim _{r \rightarrow+\infty}\left\|\widetilde{\ell}_{k_{r}}-\tilde{\ell}_{0}\right\|_{C_{1}^{0} \rightarrow \widetilde{C}^{0}}=0
$$


$\sigma \ell_{0}$ is a nonnegative linear operator, and

$$
0 \leq \sigma \ell_{0}(1)(t) \leq h(1)(t) \quad \text { for } t \in I
$$

Proof Assume that $P:=\left\{p_{1}, p_{2}, \ldots\right\}$ is a set of all polynomials with rational coefficients, then from (36) it is clear that, for the monotone linear operators $\tilde{\ell}_{k}(k \in N)$ and arbitrary $p_{j} \in P$, the inequalities

$$
\begin{aligned}
& \left|\widetilde{\ell}_{k}\left(p_{j}\right)(t)\right| \leq \int_{a}^{t}|h(1)(s)| d s\left\|p_{j}\right\|_{C_{1}^{0}} \quad \text { for } t \in I, \\
& \left|\widetilde{\ell}_{k}\left(p_{j}\right)\left(t_{2}\right)-\tilde{\ell}_{k}\left(p_{j}\right)\left(t_{1}\right)\right| \leq \int_{t_{1}}^{t_{2}}|h(1)(s)| d s\left\|p_{j}\right\|_{C_{1}^{0}} \quad \text { for } a \leq t_{1} \leq t_{2} \leq b,
\end{aligned}
$$

and

$$
\left\|\widetilde{\ell}_{k}\right\|_{C_{1}^{0} \rightarrow \widetilde{C}^{0}} \leq 2\|h(1)\|_{L}
$$

hold. Then by the Arzela-Ascoli lemma, from the sequence $\left(\tilde{\ell}_{k}\right)_{k=1}^{\infty}$ we can choose the subsequence $\left(\widetilde{\ell}_{k_{m_{1}}}\right)_{m_{1}=1}^{\infty}$ convergent on the polynomial $p_{1} \in P$, and if the subsequence of the operators $\left(\tilde{\ell}_{k_{m}}\right)_{m_{r}=1}^{\infty}$ convergent on the polynomial $p_{r} \in P$ is already chosen, then we can choose its subsequence $\left(\tilde{\ell}_{k_{m_{r+1}}}\right)_{m_{r+1}=1}^{\infty}$ of the operators convergent on the polynomial $p_{r+1} \in$ $P$. Therefore it is clear that the sequence $\left(\tilde{\ell}_{k_{m r}}\right)_{r=1}^{\infty}$ is convergent for the all polynomials from $P$. Consequently, without loss of generality, we can assume that the first subsequence $\left(\widetilde{\ell}_{k_{r}}\right)_{r=1}^{\infty}$ is convergent for arbitrary $p_{j} \in P$, and then in view of (39) and the fact that $P$ is dense in $C_{1}^{0}(I ; R)$, from the Banach-Steinhaus theorem (see [19], Theorem 3, p. 203), there follows the existence of linear operator $\tilde{\ell_{0}}: C_{1}^{0}(I ; R) \rightarrow \widetilde{C}^{0}(I ; R)$ such that condition (37) holds, where $\ell_{0}(x)(t):=\frac{d \widetilde{\ell}_{0}(x)(t)}{d t}$.

From the monotonicity of the operators $\ell_{k}$ and conditions (36) it follows that $\sigma \ell_{k}$ are the nonnegative operators, and for arbitrary $x \in C_{1}^{0}\left(I ; R_{+}\right)$we have

$$
\begin{aligned}
\sigma \int_{t_{1}}^{t_{2}} \ell_{0}(x)(s) d s & =\sigma \int_{t_{1}}^{t_{2}}\left(\ell_{0}(x)(s)-\ell_{k}(x)(s)\right) d s+\sigma \int_{t_{1}}^{t_{2}} \ell_{k}(x)(s) d s \\
& \geq-\int_{a}^{b}\left|\widetilde{\ell}_{0}^{\prime}(x)(s)-\tilde{\ell}_{k}^{\prime}(x)(s)\right| d s \\
& \geq-\left\|\tilde{\ell}_{k}-\tilde{\ell}_{0}\right\|_{C_{1}^{0} \rightarrow \widetilde{C}^{0}} \cdot\|x\|_{C_{1}^{0}} \quad \text { for } a \leq t_{1} \leq t_{2} \leq b,
\end{aligned}
$$

and

$$
\begin{aligned}
& \int_{t_{1}}^{t_{2}}\left(\sigma \ell_{0}(1)(s)-h(1)(s)\right) d s \\
& \quad=\int_{t_{1}}^{t_{2}}\left(\sigma \ell_{0}(1)(s)-\ell_{k}(1)(s)\right) d s+\int_{t_{1}}^{t_{2}}\left(\ell_{k}(1)(s)-h(1)(s)\right) d s \\
& \quad \leq \int_{a}^{b}\left|\widetilde{\ell}_{0}^{\prime}(1)(s)-\widetilde{\ell}_{k}^{\prime}(1)(s)\right| d s \leq\left\|\widetilde{\ell}_{k}-\tilde{\ell}_{0}\right\|_{C_{1}^{0} \rightarrow \widetilde{C}^{0}} \quad \text { for } a \leq t_{1} \leq t_{2} \leq b .
\end{aligned}
$$

From the last inequalities by (37) it is clear that $\sigma \ell_{0}$ is a nonnegative linear operator and inequality (38) holds. 
Now consider the problem

$$
\begin{aligned}
& u_{i}^{\left(m_{i}\right)}(t)=\lambda \sigma_{i} h_{i}\left(u_{i+1}\right)(t)+(1-\lambda)\left[F_{i}(u)(t)+q_{0 i}(t)\right] \quad(i=\overline{1, n}), \\
& u_{i}^{(j)}(b)-u_{i}^{(j)}(a)=\lambda c_{i j} \quad\left(i=\overline{1, n}, j=\overline{0, m_{i}-1}\right),
\end{aligned}
$$

where $u:=\left(u_{i}\right)_{i=1}^{n}$ and $u_{n+1}=u_{1}$. It is not difficult to verify that the following proposition is true.

Proposition 3.1 Let $m=\sum_{j=1}^{n} m_{j}, \alpha_{0}=0$, and $\alpha_{i}=\sum_{j=1}^{i} m_{j}(i=\overline{1, n})$. Then, for arbitrary $\lambda \in(0,1)$, problems (40), (41) and (16), (17) are equivalent respectively to the problems

$$
\begin{aligned}
& w_{i}^{\prime}(t)=\lambda \widetilde{\sigma}_{i} \widetilde{h}_{i}\left(u_{i+1}\right)(t)+(1-\lambda)\left[\widetilde{F}_{i}(w)(t)+\widetilde{q}_{0 i}(t)\right] \quad(i=\overline{1, m}), \\
& w_{i}(b)-w_{i}(a)=\lambda \widetilde{c}_{i} \quad(i=\overline{1, m}),
\end{aligned}
$$

and

$$
w_{i}^{\prime}(t)=\tilde{\ell}_{i}\left(w_{i+1}\right)(t), \quad w_{i}(b)-w_{i}(a)=0 \quad(i=\overline{1, m}),
$$

where $w:=\left(w_{j}\right)_{j=1}^{m}, w_{m+1}=w_{1}=u_{1}$. For all $i \in \overline{1, n}$, the following identities hold:

$$
\begin{array}{ll}
\widetilde{c}_{\alpha_{i-1}+j+1}=c_{i j} & \left(j=\overline{0, m_{i}-1}\right), \quad w_{\alpha_{i-1}+1} \equiv u_{i}, \quad \widetilde{q}_{0 \alpha_{i}} \equiv q_{0 i}, \\
\widetilde{F}_{\alpha_{i}}(w) \equiv F_{i}(u), \quad \widetilde{h}_{\alpha_{i}} \equiv h_{i}, & \widetilde{\ell}_{\alpha_{i}} \equiv \ell_{i}, \quad \widetilde{\sigma}_{\alpha_{i}}=\sigma_{i},
\end{array}
$$

and

$$
\widetilde{\sigma}_{j} \widetilde{h}_{j} \equiv \widetilde{\sigma}_{j} \tilde{\ell}_{j} \equiv \widetilde{I}, \quad \widetilde{F}_{j}(w) \equiv w_{j+1}, \quad \tilde{q}_{0 j} \equiv 0 \quad \text { if } j \notin\left\{\alpha_{1}, \alpha_{2}, \ldots, \alpha_{n}\right\}
$$

where $\widetilde{I}: C_{1}^{0}(I, R) \rightarrow L(I, R)$ is the identical operator. Moreover, it is clear that $\|w\|_{C_{m}^{0}}=$ $\|u\|_{C^{m_{1}, \ldots, m_{n}}}$.

Taking into account Proposition 3.1 we get the following modification of Corollary 2 of paper [20], which is formulated for the system of first-order equations.

Proposition 3.2 Let the linear nonnegative operators $h_{i}: C_{1}^{0}(I ; R) \rightarrow L(I ; R)(i=\overline{1, n})$ be such that inclusion (13) holds, and there exists a positive number $\rho_{1}$ such that, for arbitrary $\lambda \in(0,1)$, every solution $u$ of problem (40), (41) admits the estimate

$$
\|u\|_{C^{m_{1}, \ldots, m_{n}}} \leq \rho_{1} .
$$

Then problem (2), (3) has at least one solution.

Lemma 3.4 Let inclusion (13) hold and $\lambda \in(0,1)$. Then there exists the number $\rho_{0}>0$ such that an arbitrary solution $u=\left(u_{i}\right)_{i=1}^{n}$ of problem (1), (41) admits to the estimate

$$
\|u\|_{C^{m_{1}, \ldots, m_{n}}} \leq \rho_{0} \sum_{i=1}^{n}\left(\mu\left(u_{i}\right)+\sum_{j=0}^{m_{i}-1}\left|c_{i j}\right|+\left\|q_{i}\right\|_{L}\right),
$$


where $\mu\left(u_{i}\right)=\min \left\{\left|u_{i}(t)\right|: t \in I\right\}$, provided that inequalities (15) hold for given $\sigma_{i} \in\{-1,1\}$ $(i=\{1, n\})$.

Proof Assume that Lemma 3.4 is not true. Then, for all $k \in N$, there exist the monotone operators $\ell_{i k}: C_{1}^{0}(I ; R) \rightarrow L(I ; R)(i=\overline{1, n})$, the functions $q_{k}:=\left(q_{i k}\right)_{i=1}^{n} \in L\left(I ; R^{n}\right)$, and the numbers $c_{i j k} \in R\left(i=\overline{1, n}, j=\overline{0, m_{i}-1}\right)$ such that

$$
0 \leq \sigma_{i} \ell_{i k}(1)(t) \leq h_{i}(1)(t), \quad \int_{a}^{b} \ell_{i k}(1)(s) d s \neq 0 \quad(i=\overline{1, n})
$$

and the problem

$$
\begin{aligned}
& u_{i k}^{\left(m_{i}\right)}(t)=\ell_{i k}\left(u_{i+1 k}\right)(t)+q_{i k}(t) \quad(i=\overline{1, n}), \\
& u_{i k}^{(j)}(b)-u_{i k}^{(j)}(a)=\lambda c_{i j k} \quad\left(i=\overline{1, n}, j=\overline{0, m_{i}-1}\right),
\end{aligned}
$$

where $u_{n+1 k}:=u_{1 k}$, has such a solution $u_{k}:=\left(u_{i k}\right)_{i=1}^{n}$ that

$$
\left\|u_{k}\right\|_{C^{m_{1}, \ldots, m_{n}}} \geq k \sum_{i=1}^{n}\left(\mu\left(u_{i k}\right)+\sum_{j=0}^{m_{i}-1}\left|c_{i j k}\right|+\left\|q_{i k}\right\|_{L}\right) .
$$

Then, if we suppose that $v_{k}:=\left(v_{i k}\right)_{i=1}^{n}$, where $v_{i k}(t)=u_{i k}(t) /\left\|u_{i k}\right\|_{C^{m_{1}, \ldots, m_{n}},}, \tilde{q}_{i k}(t)=q_{i k}(t) /$

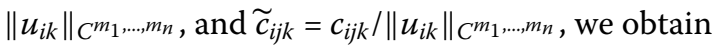

$$
\left\|v_{k}\right\|_{C^{m_{1}, \ldots, m_{n}}}=1, \quad \sum_{i=1}^{n}\left(\mu\left(v_{i k}\right)+\sum_{j=0}^{m_{i}-1}\left|\widetilde{c}_{i j k}\right|+\left\|\widetilde{q}_{i k}\right\|_{L}\right) \leq \frac{1}{k},
$$

and almost everywhere on $I$ the equalities

$$
\begin{aligned}
& v_{i k}^{\left(m_{i}\right)}(t)=\ell_{i k}\left(v_{i+1 k}\right)(t)+\widetilde{q}_{i k}(t) \quad(i=\overline{1, n}), \\
& v_{i k}^{(j)}(b)-v_{i k}^{(j)}(a)=\lambda \widetilde{c}_{i j k} \quad\left(i=\overline{1, n}, j=\overline{0, m_{i}-1}\right)
\end{aligned}
$$

hold where $v_{n+1 k}:=v_{1 k}$, from which due to (44) and (45) we obtain

$$
\left|v_{i k}^{\left(m_{i}\right)}(t)\right| \leq h_{i}(1)(t)+\left|\widetilde{q}_{i k}(t)\right| \quad(i=\overline{1, n}) \text { for } t \in I .
$$

According to $\left\|v_{k}\right\|_{C^{m_{1}, \ldots, m_{n}}}=1$, (45), and (47), for arbitrarily fixed $i_{0} \in \overline{1, n}$, sequences $\left(v_{i_{0}}^{(j)}\right)_{k=1}^{+\infty}\left(j=\overline{0, m_{i_{0}}-1}\right)$ are uniformly bounded and equicontinuous on $I$. By the ArzelaAscoli lemma, without loss of generality, it can be assumed that these sequences are uniformly convergent on $I$. Therefore, for arbitrary $i_{0} \in\{1, \ldots, n\}$, there exists such a function $v_{i_{0}} \in \widetilde{C}^{m_{i_{0}-1}}(I ; R)$ that $v_{i_{0}}^{(j)}=\lim _{k \rightarrow+\infty} v_{i_{0} k}^{(j)}\left(j=\overline{0, m_{i}-1}\right)$. Then from (45) and (46) it follows that the function $v:=\left(v_{i}\right)_{i=1}^{n} \in C^{m_{1}, \ldots, m_{n}}\left(I ; R^{n}\right)$ admits to conditions (17),

$$
\lim _{k \rightarrow+\infty}\left\|v_{k}-v\right\|_{C^{m_{1}, \ldots, m_{n}}}=0, \quad\|v\|_{C^{m_{1}, \ldots, m_{n}}}=1,
$$

and

$$
\mu\left(v_{i}\right)=0 \quad(i=\overline{1, n}) .
$$


Also due to (44) all the assumptions of Lemma 3.3 hold, and then without loss of generality we can assume that the sequence of operators $\left(\ell_{i k}\right)_{k=1}^{\infty}$ is convergent for arbitrary fixed $i \in\{1, \ldots, n\}$, and there exist the monotone operators $\ell_{i}: C_{1}^{0}(I ; R) \rightarrow L(I ; R)$ such that if the operators $\tilde{\ell}_{i k}, \tilde{\ell}_{i}: C_{1}^{0}(I ; R) \rightarrow \widetilde{C}^{0}(I ; R)$ are defined as

$$
\tilde{\ell}_{i k}(x)(t):=\int_{a}^{t} \ell_{i k}(x)(s) d s, \quad \tilde{\ell}_{i}(x)(t):=\int_{a}^{t} \ell_{i}(x)(s) d s,
$$

then

$$
\lim _{k \rightarrow+\infty}\left\|\widetilde{\ell}_{i k}-\widetilde{\ell}_{i}\right\|_{C_{1}^{0} \rightarrow \widetilde{C}^{0}}=0 \quad(i=\overline{1, n})
$$

$\sigma_{i} \ell_{i}$ is a nonnegative linear operator, and

$$
0 \leq \sigma_{i} \ell_{i}(1)(t) \leq h_{i}(1)(t) \quad(i=\overline{1, n}) \text { for } t \in I .
$$

Thus from the inequalities

$$
\begin{aligned}
& \left|\int_{a}^{t}\left(\ell_{i}\left(v_{i+1}\right)(s)-\ell_{i k}\left(v_{i+1 k}\right)(s)\right) d s\right| \\
& \quad \leq\left|\int_{a}^{t}\left(\ell_{i}\left(v_{i+1}\right)(s)-\ell_{i k}\left(v_{i+1}\right)(s)\right) d s\right|+\left|\int_{a}^{t} \ell_{i k}\left(v_{i+1}-v_{i+1 k}\right)(t) d s\right| \\
& \quad \leq\left\|\tilde{\ell}_{i k}-\tilde{\ell}_{i}\right\|_{C_{1}^{0} \rightarrow \tilde{C}^{0}} \cdot\left\|v_{i+1}\right\|_{C_{1}^{0}}+\left\|h_{i}(1)\right\|_{L} \cdot\left\|v_{i+i}-v_{i+1 k}\right\|_{C_{1}^{0}},
\end{aligned}
$$

by (48) and (50) it follows that

$$
\lim _{k \rightarrow+\infty} \int_{a}^{t} \ell_{i k}\left(v_{i+1 k}\right)(s) d s=\int_{a}^{t} \ell_{i}\left(v_{i+1}\right)(s) d s \quad(i=\overline{1, n})
$$

uniformly on $I$.

Therefore if we integrate the equations of system (46) from $a$ to $t$ and pass to the limit as $k \rightarrow+\infty$, due to conditions (45), (46), (48), and (52), we find that $v$ is a solution of problem (16), (17). On the other hand, from the inclusion $h \in P(I)$ and conditions (51), it follows that problem (16), (17) has only the zero solution $v \equiv 0$ if $\ell_{i}(1) \not \equiv 0(i=\overline{1, n})$. But if there exists $i_{0} \in\{1, \ldots, n\}$ such that $\ell_{i_{0}}(1) \equiv 0$, then from the $i_{0}$ th equation of system (16) it follows that $v_{i_{0}}^{\left(m_{i_{0}}\right)} \equiv 0$. Then from (17) we have $v_{i_{0}} \equiv$ Const, and due to (49) it is clear that $v_{i_{0}} \equiv 0$, and from the $\left(i_{0}-1\right)$ th equation of system (16), it follows that $v_{i_{0}-1}^{\left(m_{i_{0}-1}\right)} \equiv 0$. After analogous $n-1$ steps we get that $v_{i} \equiv 0(i=\overline{1, n})$. Therefore we get the contradiction with the second equality of (48), i.e., our assumption is invalid and estimation (43) holds.

\section{Proof of main results}

Proof of Theorem 2.1 It is known from the general theory of boundary value problems for the functional differential equations that problem (1), (3) has Fredholm's property (see [14]), and therefore our problem is uniquely solvable iff the homogeneous problem (16), 
(17) has only the trivial solution. Assume to the contrary that problem (16), (17) has the nontrivial solution $\left(v_{i}\right)_{i=1}^{n}$, and introduce the notations:

$$
m_{n+1}:=m_{1}, \quad v_{n+1}:=v_{1}, \quad \ell_{n+1}:=\ell_{1} .
$$

Let there exist $r \in\{\overline{1, n}\}$ such that $v_{r+1} \equiv$ Const. Then from (16) and (17) we get $v_{r+1}(t) \int_{a}^{b} \ell_{r}(1)(s) d s \equiv 0$. From the last equality and conditions (10), (16), it is obvious that $v_{r+1} \equiv 0$ and $v_{r}^{\left(m_{r}\right)} \equiv 0$. But from the identity $v_{r}^{\left(m_{r}\right)} \equiv 0$, due to conditions (17), we get that $v_{r} \equiv$ Const. After analogous $n-1$ steps we get that $v_{i} \equiv 0$ for all $i \in \overline{1, n}$, which is the contradiction with our assumption, i.e.,

$$
v_{i} \not \equiv \text { Const } \quad(i=\overline{1, n}) .
$$

Now define for all $i \in \overline{1, n}$ the numbers $\alpha_{i}, \beta_{i}, t_{1 i}, t_{2 i}, t_{1 i}^{\prime}, t_{2 i}^{\prime} \in[a, b]$ and $\sigma_{i} \in\{-1,1\}$ by the equalities

$$
\begin{aligned}
& v_{i}^{\left(m_{i}-1\right)}\left(t_{1 i}\right)=\min _{t \in[a, b]} v_{i}^{\left(m_{i}-1\right)}(t), \quad v_{i}^{\left(m_{i}-1\right)}\left(t_{2 i}\right)=\max _{t \in[a, b]} v_{i}^{\left(m_{i}-1\right)}(t), \\
& v_{i}\left(t_{1 i}^{\prime}\right)=\min _{t \in[a, b]} v_{i}(t), \quad v_{i}\left(t_{2 i}^{\prime}\right)=\max _{t \in[a, b]} v_{i}(t), \\
& \alpha_{i}:=\min \left\{t_{1 i}, t_{2 i}\right\}, \quad \beta_{i}:=\max \left\{t_{1 i}, t_{2 i}\right\}, \\
& \sigma_{i}=\operatorname{sgn}\left(v_{i}^{\left(m_{i}-1\right)}\left(\alpha_{i}\right)-v_{i}^{\left(m_{i}-1\right)}\left(\beta_{i}\right)\right),
\end{aligned}
$$

and notice that due to (17) and (54) the functions $v_{i}^{\left(m_{i}-1\right)}(i=\overline{1, n})$ change the sign, and then in view of (34) we have

$$
0<\Delta\left(v_{i}^{\left(m_{i}-1\right)}\right)=\left|v_{i}^{\left(m_{i}-1\right)}\left(\alpha_{i}\right)-v_{i}^{\left(m_{i}-1\right)}\left(\beta_{i}\right)\right| \quad(i=\overline{1, n}) .
$$

Therefore in view of (16) and (55) we get

$$
\begin{aligned}
0 & <\Delta\left(v_{i}^{\left(m_{i}-1\right)}\right)=\sigma_{i}\left(v_{i}^{\left(m_{i}-1\right)}\left(\alpha_{i}\right)-v_{i}^{\left(m_{i}-1\right)}\left(\beta_{i}\right)\right) \\
& =\sigma_{i}\left(v_{i}^{\left(m_{i}-1\right)}\left(\alpha_{i}\right)-v_{i}^{\left(m_{i}-1\right)}(a)\right)+\sigma_{i}\left(v_{i}^{\left(m_{i}-1\right)}(b)-v_{i}^{\left(m_{i}-1\right)}\left(\beta_{i}\right)\right) \\
& =\sigma_{i} \int_{a}^{\alpha_{i}} \ell_{i}\left(v_{i+1}\right)(s) d s+\sigma_{i} \int_{\beta_{i}}^{b} \ell_{i}\left(v_{i+1}\right)(s) d s, \\
0 & <\Delta\left(v_{i}^{\left(m_{i}-1\right)}\right)=\sigma_{i}\left(v_{i}^{\left(m_{i}-1\right)}\left(\alpha_{i}\right)-v_{i}^{\left(m_{i}-1\right)}\left(\beta_{i}\right)\right) \\
& =-\sigma_{i} \int_{\alpha_{i}}^{\beta_{i}} \ell_{i}\left(v_{i+1}\right)(s) d s .
\end{aligned}
$$

Assume that $\sigma_{i} \ell_{i}$ is a nonnegative operator, then from equalities (56) by Lemma 3.2 with $\ell=\sigma_{i} \ell_{i}, \sigma=1$, we have

$$
\begin{aligned}
& 0<\Delta\left(v_{i}^{\left(m_{i}-1\right)}\right) \leq v_{i+1}\left(t_{2 i+1}^{\prime}\right)\left(\int_{a}^{\alpha_{i}}\left|\ell_{i}(1)(s)\right| d s+\int_{\beta_{i}}^{b}\left|\ell_{i}(1)(s)\right| d s\right), \\
& 0<\Delta\left(v_{i}^{\left(m_{i}-1\right)}\right) \leq-v_{i+1}\left(t_{1 i+1}^{\prime}\right) \int_{\alpha_{i}}^{\beta_{i}}\left|\ell_{i}(1)(s)\right| d s .
\end{aligned}
$$


Now if we multiply the least two expressions by the well-known inequality $A \cdot B \leq(A+$ $B)^{2} / 4$ if $A \geq 0, B \geq 0$, and (34), we obtain

$$
0<\Delta\left(v_{i}^{\left(m_{i}-1\right)}\right) \leq \frac{1}{4} \int_{a}^{b}\left|\ell_{i}(1)(s)\right| d s \Delta\left(v_{i+1}\right) .
$$

It is not difficult to verify that inequality (57) holds even when the operator $\sigma_{i} \ell_{i}$ is nonpositive. On the other hand, due to (17) and (54), all the assumptions of Lemma 3.1 hold for the functions $v_{i}$ with $m=m_{i+1}-1$; and consequently, (57) by (33) implies

$$
0<\Delta\left(v_{i}^{\left(m_{i}-1\right)}\right)<\frac{(b-a)^{m_{i+1}-1}}{4 T_{m_{i+1}-1}} \int_{a}^{b}\left|\ell_{i}(1)(s)\right| d s \Delta\left(v_{i+1}^{\left(m_{i+1}-1\right)}\right) .
$$

Finally, if we multiply inequalities (58) for all $i \in \overline{1, n}$ and take into account notations (53), we get the contradiction to condition (11). Therefore our assumption is invalid and $v_{i} \equiv 0$ $(i=\overline{1, n})$, which definitely proves our theorem.

Proof of Theorem 2.2 First of all, notice that in view of inclusion (13) inequalities (14) hold, and then there exists $r_{1}>r_{0}$ such that

$$
r_{1}\left\|h_{i}(1)\right\|_{L}>\left|c_{i m_{i}-1}\right| \quad(i=\overline{1, n}) .
$$

Let now $\lambda \in(0,1)$ be an arbitrary fixed number and $u:=\left(u_{i}\right)_{i=1}^{n}$ be a solution of problem (40), (41) and show that

$$
\mu\left(u_{i}\right)=\min \left\{\left|u_{i}(t)\right|: t \in I\right\} \leq r_{1} \quad(i=\overline{1, n}) .
$$

Assume to the contrary that there exists $i \in\{1, \ldots, n\}$ such that $\left|u_{i+1}(t)\right|>r_{1}$ on $I$. Then there exist $\widetilde{\sigma}_{i} \in\{-1,1\}$ such that

$$
\tilde{\sigma}_{i} \operatorname{sgn} h_{i}\left(x_{i+1}\right)(t) \geq 0 \quad \text { on } I,
$$

and due to (20), (21), (40), (41), and (59), we obtain the contradiction

$$
\begin{aligned}
\left|c_{i m_{i}-1}\right| \geq & \lambda c_{i m_{i}-1} \sigma_{i} \widetilde{\sigma}_{i}=\sigma_{i} \widetilde{\sigma}_{i}\left(u_{i}^{\left(m_{i}-1\right)}(b)-u_{i}^{\left(m_{i}-1\right)}(a)\right) \\
= & \sigma_{i} \int_{a}^{b} u_{i}^{\left(m_{i}\right)}(s) \widetilde{\sigma}_{i} d s=\lambda \int_{a}^{b}\left|h_{i}\left(u_{i+1}\right)(s)\right| d s \\
& +(1-\lambda) \sigma_{i} \int_{a}^{b}\left[F_{i}(u)(s)+q_{0 i}(s)\right] \widetilde{\sigma}_{i} d s \geq \lambda r_{1}\left\|h_{i}(1)\right\|_{L} \\
& +(1-\lambda)\left(\int_{a}^{b} g_{i}(s) d s-\left|\int_{a}^{b} q_{0 i}(s) d s\right|\right)>\lambda\left|c_{i m_{i}-1}\right|+(1-\lambda)\left|c_{i m_{i}+1}\right|=\left|c_{i m_{i}-1}\right|
\end{aligned}
$$

which proves (60). 
Let $\rho_{0}$ be a number defined in Lemma 3.4, then due to condition (18) there exists such a constant $\rho_{1}>r_{0}$ that

$$
\begin{aligned}
& \rho_{0} \sum_{i=1}^{n}\left(\mu\left(u_{i}\right)+\sum_{j=0}^{m_{i}-1}\left|c_{i j}\right|+3\|\alpha\|_{L}+\left\|q_{0 i}\right\|_{L}+\int_{a}^{b} \eta_{i}(s, \rho) d s\right) \\
& <\rho \quad \text { for } \rho \geq \rho_{1},
\end{aligned}
$$

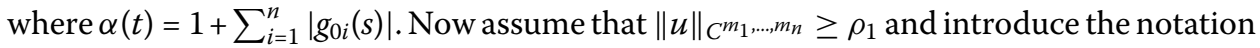

$$
v_{i}(t):=\frac{\sigma_{i} F_{i}(u)(t) \operatorname{sgn} h_{i}\left(u_{i+1}\right)(t)+\alpha(t)}{\left|h_{i}\left(u_{i+1}\right)(t)\right|+\eta_{i}\left(t,\|u\|_{\left.C^{m_{1}, \ldots, m_{n}}\right)+2 \alpha(t)}\right.} \quad \text { for } t \in I .
$$

Then, in view of conditions (19), we have

$$
0<v_{i}(t)<1 \quad(i=\overline{1, n}) \text { on } I .
$$

On the other hand it is not difficult to verify that $u$ is a solution also of the system (1), with

$$
\begin{aligned}
& \ell_{i}(x)(t)=\sigma_{i}\left[\lambda+(1-\lambda) v_{i}(t)\right] h_{i}(x)(t), \\
& q_{i}(t)=(1-\lambda)\left[\left(v_{i}(t)\left[2 \alpha(t)+\eta_{i}\left(t,\|u\|_{C^{m_{1}, \ldots, m_{n}}}\right)\right]-\alpha(t)\right) \sigma_{i} \operatorname{sgn} h_{i}\left(u_{i+1}\right)(t)+q_{0 i}(t)\right],
\end{aligned}
$$

where due to inequalities (62) the following estimations are valid:

$$
\begin{aligned}
& 0 \leq \sigma_{i} \ell_{i}(1)(t) \leq h_{i}(1)(t), \\
& \left|q_{i}(t)\right| \leq 3 \alpha(t)+\left|q_{0 i}(t)\right|+\eta_{i}\left(t,\|u\|_{C^{m_{1}, \ldots, m_{n}}}\right)
\end{aligned}
$$

on $I$. Then, for the function $u$, as for a solution of problem (1), (41), by Lemma 3.4 we get the estimation

$$
\begin{aligned}
& \|u\|_{C^{m_{1}, \ldots, m_{n}}} \\
& \leq \rho_{0} \sum_{i=1}^{n}\left(\mu\left(u_{i}\right)+\sum_{j=0}^{m_{i}-1}\left|c_{i j}\right|+3\|\alpha\|_{L}+\left\|q_{0 i}\right\|_{L}+\int_{a}^{b} \eta_{i}\left(s,\|u\|_{C^{m_{1}, \ldots, m_{n}}}\right) d s\right),
\end{aligned}
$$

which due to the assumption $\|u\|_{C^{m_{1}, \ldots, m_{n}}} \geq \rho_{1}$ contradicts (61). Therefore our assumption is invalid and estimation (42) holds, and then from Proposition 3.2 the solvability of problem (2), (3) follows.

Proof of Corollary 2.2 In view of Corollary 2.1, from conditions (14) and (23), it follows that, for arbitrary monotone operators $\ell_{i}: C(I ; R) \rightarrow L(I ; R)(i=\overline{1, n})$ which admit to inequalities (15), problem (16), (17) has only the zero solution, and then inclusion (13) holds. Therefore all the assumptions of Theorem 2.2 are fulfilled, and then problem (2), (3) is solvable.

Proof of Corollary 2.3 Let $h_{i}(x)(t)=\widetilde{h}_{i}(t) x\left(\tau_{i}(t)\right)$, then from conditions (25) and Theorem 2.1 it follows that inclusion (13) holds, and

$$
\operatorname{sgn} h_{i}(x)(t)=\operatorname{sgn} x\left(\tau_{i}(t)\right) \quad(i=\overline{1, n}) \text { a.e. on } I .
$$


Also, it is clear that due to (26), for arbitrary $x \in C^{m_{1}, \ldots, m_{n}}\left(I ; R^{n}\right)$, the conditions

$$
\begin{aligned}
& g_{i}(t) \leq \sigma_{i} f_{i}\left(t, x_{i+1}\left(\tau_{i}(t)\right)\right) \operatorname{sgn} x_{i+1}\left(\tau_{i}(t)\right) \\
& \quad \text { for } t \in\left\{s \in I:\left|x_{i+1}\left(\tau_{i}(s)\right)\right| \geq r_{0}\right\}(i=\overline{1, n})
\end{aligned}
$$

are fulfilled. On the other hand, if

$$
\widetilde{g}_{i}(t):=\max \left\{\left|f_{i}(t, y)\right|:|y| \leq r_{0}\right\}, \quad g_{0 i}(t):=\min \left\{-\widetilde{g}_{i}(t), g_{i}(t)\right\} \quad(i=\overline{1, n}),
$$

then, in view of (26) and the fact that the functions $\eta_{i}$ are nondecreasing in the second argument, we obtain

$$
\begin{aligned}
& g_{0 i}(t) \leq \sigma_{i} f_{i}\left(t, x_{i+1}\left(\tau_{i}(t)\right)\right) \operatorname{sgn} x_{i+1}\left(\tau_{i}(t)\right) \leq \widetilde{h}_{i}(t)\left|x_{i+1}\left(\tau_{i}(t)\right)\right|+\widetilde{g}_{i}(t)+\eta_{i}\left(t,\|x\|_{\left.C^{m_{1}, \ldots, m_{n}}\right)}\right. \\
& \quad \text { for }\|x\|_{C^{m_{1}, \ldots, m_{n}}} \geq r_{0}, t \in I(i=\overline{1, n}),
\end{aligned}
$$

and due to (18) the following equalities hold:

$$
\lim _{\rho \rightarrow+\infty} \frac{1}{\rho} \int_{a}^{b}\left(\widetilde{g}_{i}(s)+\eta_{i}\left(s,\|x\|_{C^{m_{1}, \ldots, m_{n}}}\right)\right) d s=0 \quad(i=\overline{1, n}) .
$$

Consequently, if we introduce the notations $F_{i}(x)(t)=f_{i}\left(t, x_{i+1}\left(\tau_{i}(t)\right)\right)$, we get that all the assumptions of Theorem 2.2 are valid, from which the validity of our corollary immediately follows.

Proof of Theorem 2.3 First note that from conditions (25), analogously as in the proof of previous Corollary 2.3, validity of inclusion (13) with $h_{i}(x)=\widetilde{h}_{i}(t) x\left(\tau_{i}(\cdot)\right)$ follows.

Also, for arbitrary $r \geq 0$, from condition (29), (30) and the fact that

$$
\operatorname{sgn}\left(x-(-1)^{j} r\right)=\operatorname{sgn} x \quad \text { if }(-1)^{j} x>r(j=0,1),
$$

we have

$$
\begin{aligned}
\sigma_{i} f_{i}\left(t,(-1)^{j} r\right) \operatorname{sgn} x & \leq \widetilde{h}_{0 i}(t) \beta_{i}(x, r)+\sigma_{i} f_{i}\left(t,(-1)^{j} r\right) \operatorname{sgn} x \leq \sigma_{i} f_{i}(t, x) \operatorname{sgn} x \\
& \leq \widetilde{h}_{i}(t)(|x|+r)+\sigma_{i} f_{i}\left(t,(-1)^{j} r\right) \operatorname{sgn} x \quad \text { for } t \in I,(-1)^{j} x>r .
\end{aligned}
$$

On the other hand, due to the conditions $f_{i}(t, 0) \equiv 0(i=\overline{1, n})$ and (30), from (29) it follows that

$$
\sigma_{i} f_{i}\left(t,(-1)^{j} r\right) \operatorname{sgn} x \geq 0 \quad \text { for } t \in I,(-1)^{j} x>r .
$$

From the last two inequalities and (21) it is clear that all the assumptions of Corollary 2.3 hold when the functions $g_{i}$ are defined by (31) and $\eta_{i}(t, x)=\widetilde{h}_{i}(t) r+\max \{|f(t, r)|,|f(t,-r)|\}$ for $t \in I,|x|>r$. Therefore it remains to prove that problem (24), (3) has no more than one solution. 
Let $\widetilde{u}_{1}:=\left(u_{1 i}\right)_{i=1}^{n}$ and $\widetilde{u}_{2}:=\left(u_{2 i}\right)_{i=1}^{n}$ be the arbitrary solutions of problem (24), (3), and $u:=\widetilde{u}_{1}-\widetilde{u}_{2}$. Then the function $u$ admits to conditions (17), and from (29) we obtain that, for all $i=\overline{1, n}$, the inequalities

$$
\begin{aligned}
& \widetilde{h}_{0 i}(t) \beta_{i}\left(\widetilde{u}_{1 i+1}(t), \widetilde{u}_{2 i+1}(t)\right) \\
& \quad \leq \sigma_{i} u_{i}^{\left(m_{i}\right)}(t) \operatorname{sgn} u_{i+1}\left(\tau_{i}(t)\right) \leq \widetilde{h}_{i}(t)\left|u_{i+1}\left(\tau_{i}(t)\right)\right|
\end{aligned}
$$

hold on $I$. Now, if we assume that there exists $i_{0} \in\{1, \ldots, n\}$ such that $\left|u_{i_{0}+1}(t)\right|>0$ on $I$, then $\operatorname{sgn} u_{i_{0}+1}\left(\tau_{i_{0}}(t)\right)=\operatorname{sgn} u_{i_{0}+1}(a)$, and also due to (30) we obtain $\beta_{0 i_{0}}:=\min \left\{\beta_{i_{0}}\left(\widetilde{u}_{1 i_{0}+1}(t)\right.\right.$, $\left.\left.\widetilde{u}_{2 i_{0}+1}(t)\right): t \in I\right\}>0,\left\|\widetilde{h}_{0 i_{0}}\right\|_{L}>0$. Therefore from (17) and (63) we get the contradiction

$$
\begin{aligned}
0 & =\sigma_{i_{0}}\left(u_{i_{0}}^{\left(m_{i_{0}}-1\right)}(b)-u_{i_{0}}^{\left(m_{i_{0}}-1\right)}(a)\right) \operatorname{sgn} u_{i_{0}+1}(a) \\
& =\sigma_{i_{0}} \int_{a}^{b} u_{i_{0}}^{\left(m_{i_{0}}\right)}(s) \operatorname{sgn} u_{i_{0}+1}\left(\tau_{i_{0}}(s)\right) d s \geq \beta_{0 i_{0}}\left\|\widetilde{h}_{0 i_{0}}\right\|_{L}>0 \quad \text { a.e. on } I,
\end{aligned}
$$

which shows that

$$
\min \left\{\left|u_{i}(t)\right|: t \in I\right\}=0 \quad(i=\overline{1, n})
$$

Suppose that

$$
p_{i}(t)= \begin{cases}u_{i}^{\left(m_{i}\right)}(t) / u_{i+1}\left(\tau_{i}(t)\right) & \text { if } u_{i+1}\left(\tau_{i}(t)\right) \neq 0, \\ 0 & \text { if } u_{i+1}\left(\tau_{i}(t)\right)=0,\end{cases}
$$

then $u$ is a solution of the linear homogeneous problem (16), (17) with $\ell_{i}(x)(t)=$ $p_{i}(t) x\left(\tau_{i}(t)\right)$, which due to condition (63) admits to the inequalities

$$
0 \leq \sigma_{i} \ell_{i}(1)(t) \leq h_{i}(1)(t) \quad(i=\overline{1, n}) \text { for } t \in I .
$$

Let $p_{i} \not \equiv 0(i=\overline{1, n})$, then in view of the inclusion $h \in P(I)$ we obtain $u \equiv 0$. Now assume that there exists $i_{0} \in\{1, \ldots, n\}$ such that $p_{i_{0}} \equiv 0$. Then from (16) the identity $u_{i_{0}}^{\left(m_{i_{0}}\right)} \equiv 0$ follows, and in view of conditions (17) and (64) we get $u_{i_{0}} \equiv 0$, from which on the basis of (16) it follows that $u_{i_{0}-1}^{\left(m_{i_{0}-1}\right)} \equiv 0$. After analogous $n-1$ steps we get that $u \equiv 0$, and therefore $\widetilde{u}_{1} \equiv \widetilde{u}_{2}$.

\section{Acknowledgements}

The research was supported by RVO: 67985840 .

Funding

Not applicable.

\section{Availability of data and materials}

Data citations (such as a DOI, URL, MR numbr, ...) when they exist are included in the reference list.

\section{Competing interests}

The authors declare that they have no competing interests.

\section{Authors' contributions}

SM contributed with the results on linear problem and partly on nonlinear problem. BP contributed partly with the results on nonlinear problem and principally in preparation of the manuscript. All authors read and approved the final manuscript. 


\section{Publisher's Note}

Springer Nature remains neutral with regard to jurisdictional claims in published maps and institutional affiliations.

Received: 16 February 2020 Accepted: 20 May 2020 Published online: 05 June 2020

\section{References}

1. Lasota, A., Opial, Z.: Sur les solutions periodiques des equations differentielles ordinaires. Ann. Pol. Math. 16, 69-94 (1964). https://doi.org/10.4064/ap-16-1-69-94

2. Mukhigulashvili, S., Lomtatidze, A.: On periodic solutions of second order functional differential equations. Mem. Differ. Equ. Math. Phys. 5, 125-126 (1995). http://eprints.liauni.edu.ge/id/eprint/4106

3. Mukhigulashvili, S.: On periodic solutions of second order functional differential equations. Ital. J. Pure Appl. Math. 20, 29-50 (2006). http://ijpam.uniud.t//journal/abstracts.htm

4. Mukhigulashvili, S.: On a periodic boundary value problem for third order linear functional differential equations. Nonlinear Anal. 66, 527-535 (2007). https://doi.org/10.1016/j.na.2005.11.046

5. Hakl, R., Mukhigulashvili, S.: On a periodic boundary value problem for third order linear functional differentia equations. Mem. Differ. Equ. Math. Phys. 41, 27-42 (2007). www.emis.de/journals/MDEMP/vol41/vol41-2.pdf

6. Hakl, R., Mukhigulashvili, S.: Periodic boundary value problem for functional differential equations of higher order. Georgian Math. J. 16, 651-665 (2009). https://doi.org/10.1515/GMJ.2009.651

7. Hakl, R., Mukhigulashvili, S.: On one estimate for the periodic functions. Georgian Math. J. 12, 97-114 (2005). https://doi.org/10.1515/GMJ.2005.97

8. Bravyi, E.: On the solvability of the periodic boundary value problem for a linear functional differential equation Vestn. Udmurtsk. Univ. Mat. Mekh. Komp. Nauki 3, 12-24 (2009). http://mi.mathnet.ru/eng/vuu/y2009/i3/p12

9. Bravyi, E.: On the solvability of linear boundary value problems for functional differential equations with intermediate derivatives. Funct. Differ. Equ. 18, 101-110 (2011). MR2894317

10. Bravyi, E.: On the best constants in the solvability conditions for the periodic boundary value problem for higher-order functional differential equations. Differ. Equ. 48, 779-786 (2012). https://doi.org/10.1134/S001226611206002X

11. Bravyi, E.: Solvability of the periodic problem for higher-order linear functional differential equations. Differ. Equ. 51, 571-585 (2015). https://doi.org/10.1134/S0012266115050018

12. Bravyi, E.: On positive periodic solutions of linear second order functional differential equations. Georgian Math. J. 24, 29-39 (2016). https://doi.org/10.1515/gmj-2016-0069

13. Bravyi, E:: On periods of non-constant solutions to functional differential equations. Electron. J. Qual. Theory Differ. Equ. 2017, 14 (2017). https://doi.org/10.14232/ejatde.2017.1.14

14. Domoshnitsky, A.: Sign properties of Green's matrices of periodic and some other problems for system of functional-differential equations. Funct. Differ. Equ. 2, 39-57 (1997). MR1386058

15. Domoshnitsky, A., Hakl, R., Sremr, J.: Component-wise positivity of solutions to periodic boundary value problem for linear functional differential systems. J. Inequal. Appl. 2012, 112 (2012). https://doi.org/10.1186/1029-242X-2012-112

16. Mukhigulashvili, S.: On a periodic boundary value problem for cyclic feedback type linear functional differential systems. Arch. Math. 87, 255-260 (2006). https://doi.org/10.1007/s00013-006-1621-1

17. Grytsay, I., Mukhigulashvili, S.: An optimal condition for the uniqueness of periodic solution for linear functional differential systems. Electron. J. Qual. Theory Differ. Equ. 2009, 59 (2009). https://doi.org/10.14232/ejqtde.2009.1.59

18. Mukhigulashvili, S., Půza, B.: On a periodic boundary value problem for cyclic feedback type linear functional differential systems. Mem. Differ. Equ. Math. Phys. 40, 67-75 (2007). www.emis.de/journals/MDEMP/vol40/vol40-4.pdf

19. Kantorovich, L., Akilov, G.: Functional Analysis. Pergamon, Oxford (1982)

20. Kiguradze, I., Pi̊za, B.: On boundary value problems for functional differential equations. Mem. Differ. Equ. Math. Phys. 12, 106-113 (1997). Zbl 0909.34054, MR1636865

\section{Submit your manuscript to a SpringerOpen ${ }^{\circ}$ journal and benefit from:}

- Convenient online submission

- Rigorous peer review

- Open access: articles freely available online

- High visibility within the field

Retaining the copyright to your article

Submit your next manuscript at $\gg$ springeropen.com 